\title{
MAGNETO-OPTIC PROPERTIES OF SELF-TRAPPED EXCITONS IN CESIUM HALIDES
}

\author{
T. IIDA, Y. NAKAOKA, J.P. VON DER WEID* and M.A. AEGERTER* \\ Department of Physics, Osaka City University, Osaka, Japan
}

\begin{abstract}
A model for the electronic structure of self-trapped excitons in cesium halides is proposed. With applying it, reasonable interpretations are obtained for the magnetic circular polarization (MCP) of the two intrinsic emissions in CsI, and for the MCP and EPR in CsBr.
\end{abstract}

\section{Introduction}

When cesium halides at low temperatures are exposed to X-ray or UV excitation, two intrinsic emissions due to the decay of the self-trapped excitons (STE) are observed [1]. In the present work, the origin of these emissions in CsI shall be investigated in detail, since less measurements have been reported for the other cesium halides. In pure CsI, the two emissions peaked at $290 \mathrm{~nm}$ and $338 \mathrm{~nm}$ have distinct features compared with those in alkali halides; both emissions are $\pi$-polarized at low temperatures and have rather long life times, $\tau_{290}=0.1 \times 10^{-6} \mathrm{~s}$ and $\tau_{338}=0.7 \times 10^{-6} \mathrm{~s}[1]$.

Theoretical calculations from the first principle for the electronic structure of STE, at present, are restricted to lighter halide systems due to a limit of number of electrons which can be treated [2]. We set up a group-theoretical model by the semiempirical molecular orbital (MO) method and apply it to explain the magneto-optic properties of the STE in CsI. We also give a consistent interpretation for the MCP and transient EPR in CsBr.

\section{Electronic structure}

It has been widely accepted that the STE can be regarded as an electron bounded to the self-trapped hole (STH). We consider a cluster composed of a diatomic molecule of halide ions $\left(\mathrm{X}^{-}\right)_{2}$, surrounded by $12 \mathrm{Cs}^{+}$ions in $\mathrm{D}_{4 \mathrm{~h}}$ symmetry. According to Stoneham [2], the orbitals of electrons around the STH in alkali halides are constructed primarily from the orbitals of neighboring alkali ions. Being guided by this fact and inferring from the fact that the d-like and the

* Institut de Physique, Université de Neuchatel, Neuchatel, Switzerland 
s-like conduction bands are nearly degenerate and mainly composed of $6 \mathrm{~s}$ and $5 \mathrm{~d}$ states of $\mathrm{Cs}^{+}$ions, we assume that the electronic states of the cluster are described by the MO's of $\left(\mathrm{X}^{-}\right)_{2}$ and those of $6 \mathrm{~s}$ and $5 \mathrm{~d}$ (with symmetries $\left.\mathrm{d}_{x^{2}-y^{2}}, \mathrm{~d}_{z^{2}}\right)$ orbitals of $\mathrm{Cs}^{+}$ions. By symmetry consideration, we obtain two types of lower lying exciton states with different symmetries, $\Gamma_{2}^{-}$(called type I) and $\Gamma_{4}^{-}$ (called type II). By taking into account the exchange interaction $(J)$ and the spin-orbit interaction $(\lambda)$, the lowest three states are given as a splitted triplet state in both types. The upper two states of each type $\left.\left(\left.\right|^{3} \Gamma_{2}^{-}: \Gamma_{5} x\right\rangle,\left.\right|^{3} \Gamma_{2}^{-}: \Gamma_{5}^{-} y\right\rangle$ in type $\mathrm{I}$ and $\left.\left.\beta^{3} \Gamma_{4}^{-}: \Gamma_{5}^{-} x\right\rangle, \beta^{3} \Gamma_{4}^{-}: \Gamma_{5}^{-} y\right\rangle$ in type II) are degenerate and give rise to the $\pi$-polarized emissions, while the lowest state $\left({ }^{3} \Gamma_{2}^{-}: \Gamma_{1}^{-}\right\rangle$in type $I$ and $\left.\left|{ }^{3} \Gamma_{4}: \Gamma_{3}^{-}\right\rangle\right)$in type II), separated by D from the upper level, is forbidden in both cases. The type I STE, which is close to that of Fowler et al. [3], is assigned to the $338 \mathrm{~nm}$ STE and the type II is to the $290 \mathrm{~nm}$ STE in this model.

\section{Comparison with experiments}

When the magnetic field $H$ is applied along $\langle 001\rangle$, in each type of STE oriented along $H$, the lowest level [called level (1)] remains almost unchanged, and the upper degenerate level split: one [called level (2)] varies linearly in $H$ with the slope $-g_{\|} \beta$ and the other [called level (3)] varies with $g_{\|} \beta$. Then the levelcrossing may occur between level (1) and (2) in both types. The essential difference between the two types is that in type I, the levels (2) and (3) have the symmetries $X-\mathrm{i} Y$ and $X+\mathrm{i} Y$, respectively. On the other hand, in type II, they have symmetries $X+\mathrm{i} Y$ and $X-\mathrm{i} Y$, respectively.

The population of each level will be determined by six coupled rate equations. We assume that the coupled equations can be decoupled into two sets of equations for each type. In the rate equations, the tunneling processes, $P_{i j} \exp \left(-Q_{i i} \delta E_{i j}\right)[3]$, and the direct one-phonon processes $W_{i j}^{2}\left(\delta E_{i j}\right)^{3} f_{i j}\left(n_{i j}+\frac{1}{2} \pm \frac{1}{2}\right)$ are taken into account in addition to the radiative ones, where $f_{i j}$ 's are calculated using explicit forms of wave functions. The general feature of population difference is shown in fig. 1(a), where $m_{i}(H)$ is the population in the ith level and the level-crossing is assumed to occur at $H=45 \mathrm{KG}$. The observed MCP
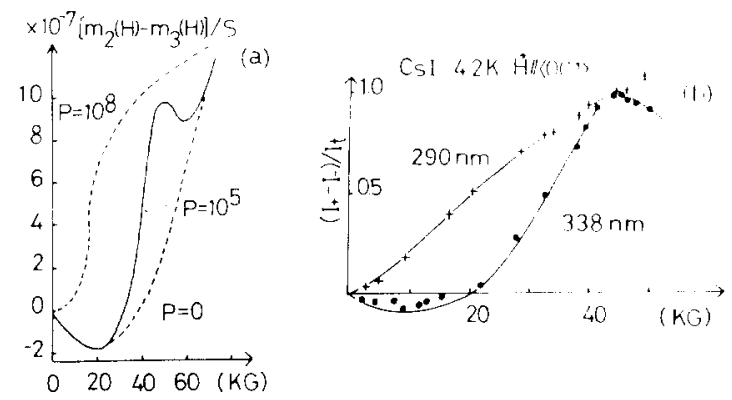

Fig. 1(a). The population difference normalized by the net supply $S$ versus the magnetic field for the sets of appropriate parameters; $V_{12}=V_{13}=V_{23}=9.5 \times 10^{4} \mathrm{~s}^{-1}, Q_{12}=Q_{13}=Q_{23}=1.95 \times 10^{4} \mathrm{eV}^{-1}$ and at $T=4.2 \mathrm{~K}$, where $V_{i j}=W_{i j}^{2} D^{3}, D$ being the zero field splitting (b) The normalized MCP of $338 \mathrm{~nm}$ and $290 \mathrm{~nm}$ emissions in Csl. The symbols + and - stand for experimental results [4]. 
Table 1

Parameter values, $\left(\gamma_{1}, \gamma_{2}, \gamma_{3}\right)$ and $\left(\gamma_{1}^{\prime}, \gamma_{2}^{\prime}, \gamma_{3}^{\prime}\right)$ are the time constants of the transient response under the microwave field and after switching off the field, respectively; $V_{i j}=W_{i j}^{2} D^{3}$; the numbers in parentheses are experimental results [7].

\begin{tabular}{|c|c|c|c|c|c|c|c|c|}
\hline & $\begin{array}{l}D \\
(\mathrm{meV})\end{array}$ & $\begin{array}{l}J \\
(\mathrm{meV})\end{array}$ & $\begin{array}{l}V_{12} \\
\left(\mathrm{~s}^{-1}\right)\end{array}$ & $V_{13}$ & $V_{23}$ & $\begin{array}{l}P_{12} \\
\left(\mathrm{~s}^{-1}\right)\end{array}$ & $P_{13}$ & $P_{23}$ \\
\hline $\begin{array}{l}\mathrm{CsBr} \\
350 \mathrm{~nm}\end{array}$ & 0.0682 & 8.95 & 2.49 & 1.12 & 2.49 & $1.1 \times 10^{4}$ & $1.1 \times 10^{4}$ & $1.1 \times 10^{7}$ \\
\hline $\begin{array}{l}\text { CsI } \\
338 \mathrm{~nm} \\
290 \mathrm{~nm}\end{array}$ & $\begin{array}{l}0.495 \\
0.576\end{array}$ & $\begin{array}{r}9.0 \\
10.5\end{array}$ & & $\begin{array}{l}4.37 \times 10^{3} \\
1.50 \times 10\end{array}$ & & & $\begin{array}{r}4.0 \times 10^{4} \\
\leq 4.0 \times 10^{2}\end{array}$ & \\
\hline & & $\begin{array}{l}Q \\
\left(\mathrm{eV}^{-1}\right)\end{array}$ & $\begin{array}{l}\gamma_{1} \\
\left(\mathrm{~s}^{-1}\right)\end{array}$ & $\gamma_{2}$ & $\gamma_{3}$ & $\begin{array}{l}\gamma_{1}^{\prime} \\
\left(\mathrm{s}^{-1}\right)\end{array}$ & $\gamma_{2}^{\prime}$ & $\gamma_{3}^{\prime}$ \\
\hline & & $\begin{array}{l}5.84 \times 10^{4} \\
9.77 \times 10^{3} \\
\approx 10^{3}\end{array}$ & $6.7 \times 10^{5}$ & $\begin{array}{l}7.0 \times 10^{4} \\
\left(\geq 10^{5}\right)\end{array}$ & $\begin{array}{l}5.4 \times 10^{3} \\
\left(5.9 \times 10^{3}\right)\end{array}$ & $6.7 \times 10^{5}$ & $\begin{array}{l}6.7 \times 10^{4} \\
\left(5 \times 10^{4}\right)\end{array}$ & $\begin{array}{l}1.3 \times 10^{3} \\
\left(2.4 \times 10^{3}\right)\end{array}$ \\
\hline
\end{tabular}

$\left(=-\left(I_{+}-I_{-}\right) / I_{t}\right)$ is approximately proportional to the population difference. Observing fig. 1(a) and the symmetry of level, we predict that the MCP is negative for low field and increases with $H$ till the extreme point in type I; while in type II, if the level-crossing is assumed to occur at $H \geqslant 50 \mathrm{kG}$, it increases smoothly with $H$ in the range $H \leqslant 50 \mathrm{kG}$. This is qualitatively consistent with the experiments (fig. 1(b)). Quantitatively, the parameters are determined as listed in table 1 and the best fit curves are shown in fig. 1(b). For the $290 \mathrm{~nm}$ emission, the level-crossing point can not be determined uniquely but it will be expected to occur in the range $50 \leq H \leq 80 \mathrm{KG}$ from the parameter analysis and the experimental results of the temperature dependence of the emission [5]. The parameters for $290 \mathrm{~nm}$ emission are determined by assuming tentatively that the level-crossing occurs at $H=53 \mathrm{kG}$, with which the curve in fig. 1(b) is computed.

In order to examine the validity of the rate equations and the procedure used to determine the parameters, we apply the model to the calculation of the transient response of EPR as well as the MCP of the lower energy emission in $\mathrm{CsBr}$, since the transient EPR experiment provides much information on the
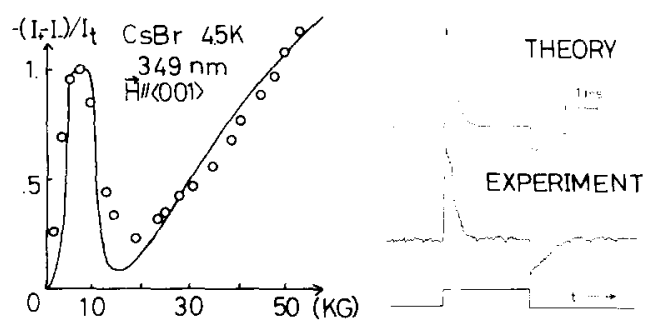

Fig. 2. Left: the normalized MCP of $350 \mathrm{~nm}$ emission in CsBr. The open circles stand for experimental result [6]; the transient responce of $\sigma_{+}$emission to the pulsed microwave excitation. The observed one is from ref. [7]. 
time constants involved in the kinetics. The best fit is obtained as shown in fig. 2 , and the corresponding parameters are listed in table 1.

The agreement with experiments is fairly good, which allows us to conclude that the model gives a reasonable description of the origin of two emissions in CsI, and that the rate equations used to determine the parameters at low temperatures describe the kinetics quite well. Further detailed study to confirm the validity of the model is in progress.

\section{References}

[1] J.P. Pellaux, Ph.D. dissertation, University of Neuchatel, Switzerland (1976).

[2] A.M. Stoneham, J. Phys. C7 (1974) 2476.

[3] W.B. Fowler, M.J. Marrone and M.N. Kabler, Phys. Rev. 8 (1973) 5909

[4] L. Falco, J.P. von der Weid and M. Aegerter, Proc. Int. Conf. on Defects in insulating crystals, Gatlinbrug (1977) 336.

[5] J.P. Pellaux, T. Iida, J.P. von der Weid and M. Aegerter, in preparation.

[6] M.N. Kabler and M.J. Marrone, private communication.

[7] Y. Mori. J.P. von der Weid and M. Aegerter. submitted to Solid State Commun. 